\title{
On hydrogen entanglements and gravitation: A Lie symmetry approach
}

\author{
JM Manale \\ The Department of Mathematical Sciences \\ University of South Africa \\ Florida, 1709, Johannesburg, Gauteng Province \\ Republic of South Africa \\ email: manaljm@unisa.ac.za, JacobManale@gmail.com
}

Received: January 6, 2021. Revised: March 17, 2021. Accepted: March 23, 2021. Published: March 26, 2021.

\begin{abstract}
We depart from the popular view on how gravitation is generated. Ours is entanglement based. For an atom, hydrogen in this case, all subatomic particles, within and outside the nuclei, participate in this eternal dance. To test the idea, we use it to determine a formula for $G$, the universal gravitational constant.

In the process, we note that the one-dimensional Schrödinger equation is not solved, as claimed. For example, existing solutions are silent on the quantum superposition principle. This we address through our modified symmetry group theoretical methods.
\end{abstract}

Keywords - Gravitation; Schrödinger equation; Symmetry Analysis; Modified Symmetries.

\section{INTRODUCTION}

$\mathbf{G}$ RAVITY or gravitation, is, perhaps, the least understood concept in the physical sciences, but yet the most important. Enormous amounts of money have been poured into understanding it, and it continues to attract more.

In this contribution, we propose a different understanding on how matter generates gravity. The one-dimensional Schrödinger equation constitutes the model equation, but begin with Newtonian mechanics for the simplicity. We use Sophus Lie's symmetry group theoretical methods, with a little modification, to solve the differential equations. This is because it is impossible to realise quantum superposition solutions through the pure Lie approach.

\section{THE QUANTUM MECHANICAL APPROACH}

The Schrödinger wave equation for the hydrogen atom with electron mass $m_{e}$ and proton mass $m_{p}$ has the form

$$
\begin{aligned}
& -\frac{\hbar^{2}}{2 m_{e}}\left[\frac{\partial^{2}}{\partial x^{2}}+\frac{\partial^{2}}{\partial y^{2}}+\frac{\partial^{2}}{\partial z^{2}}\right] \psi \\
& -\frac{\hbar^{2}}{2 m_{p}}\left[\frac{\partial^{2}}{\partial X^{2}}+\frac{\partial^{2}}{\partial Y^{2}}+\frac{\partial^{2}}{\partial Z^{2}}\right] \psi \\
& +(V-E) \psi=i \hbar \frac{\partial \psi}{\partial t} .
\end{aligned}
$$

Here $\psi=\psi(t, \mathbf{r}, \mathbf{R})$ is the probability density function, $V$ is the potential, $E$ is the energy level, $\mathbf{r}=(x, y, z)$ is the position of the electron, $\mathbf{R}=(X, Y, Z)$ is that of the proton, and $\hbar$ is the reduced Planck constant.
Our interest is in the motion of the electron and proton along the same straight path. This reduces (1) to the one-dimensional Schrödinger wave equation,

$$
-\frac{\hbar^{2}}{2 m_{e}} \frac{\partial^{2} \psi}{\partial x^{2}}-\frac{\hbar^{2}}{2 m_{p}} \frac{\partial^{2} \psi}{\partial X^{2}}+(V-E) \psi=i \hbar \frac{\partial \psi}{\partial t},
$$

where $\psi=\psi(t, x, X)$ is the probability density function, $V$ is the potential, $E$ is the energy level, $x$ is the position of the electron with mass $m_{e}$ from $X$, with $X$ being that of the proton with mass $m_{p}$ from the origin.

Since the mass of the proton is much larger than that of the proton, then the electron can be interpreted as moving around around a positive core frozen in place in the static potential field $V(x, X)$. The solution of a bound electron with $V=V(x)$ then fits this equation. That is,

$$
-\frac{\hbar^{2}}{2 m_{e}} \frac{d^{2} \phi}{d x^{2}}+(V-E) \phi=0
$$

where $\psi=\varphi(t, X) \phi(t, x)$. This equation is that of a forced particle. This then generates another equation from (2). Namely,

$$
-\frac{\hbar^{2}}{2 m_{p}} \frac{\partial^{2} \varphi}{\partial X^{2}}=i \hbar \frac{\partial \varphi}{\partial t} .
$$

Equation (4) describes the motion of a free particle, implying that the proton is moving freely.

\section{THE FORCED WAVE EQUATION (3)}

In the case of a coulombic force, we have

$$
V=-\frac{1}{4 \pi \epsilon_{0}} \frac{q Q}{x},
$$

where $q$ and $Q$ are the respective charges. This transforms (3) into

$$
\frac{\hbar^{2}}{2 m_{e}} \frac{d^{2} \phi}{d x^{2}}+\left(\frac{1}{4 \pi \epsilon_{0}} \frac{q Q}{x}+E\right) \phi=0
$$

or

$$
\frac{d^{2} \phi}{d x^{2}}+\left(\frac{m_{e} q Q}{2 \pi \epsilon_{0} \hbar^{2}} \frac{1}{x}+\frac{2 m_{e} E}{\hbar^{2}}\right) \phi=0 .
$$




\section{A. Solutions through differentiable Manifolds}

We start with a topological space $\mathcal{M}=\left(X, \mathcal{J}_{X}\right)$, a Hausdorff topology. That is, a set $X$ with a topology $\mathcal{J}_{X}$. For it to be a differentiable topological manifold, or simply a differentiable manifold, we require an atlas $\mathcal{A}$ in addition, then we have $\mathcal{D} \mathcal{M}=\left(X, \mathcal{J}_{X}, \mathcal{A}\right)$.

We now consider two points $p \in U_{p}$ and $q \in U_{q}$, such that the sets $U_{p}$ and $U_{q}$ are elements of the same manifold. We can then build the sub-topologies $\left(U_{p},\left.\mathcal{J}_{X}\right|_{U_{p}}\right)$ and $\left(U_{q},\left.\mathcal{J}_{X}\right|_{U_{q}}\right)$. That is, the topology of $X$ restricted to $U_{p}$ and $U_{q}$. A mapping $\psi_{p}$, if it exists, then maps the space $\left(U_{p},\left.\mathcal{J}_{X}\right|_{U_{p}}\right)$ into the Euclidean space $\left(\left.\mathbb{R}^{N} \mathcal{J}_{\mathbb{R}^{N}}\right|_{\psi_{p}\left(U_{p}\right)}\right)$. Similarly, $\psi_{q}$ maps $\left(U_{q},\left.\mathcal{J}_{X}\right|_{U_{q}}\right)$ into the Euclidean space $\left(\mathbb{R}^{N}, \mathcal{J}_{\left.\left.\mathbb{R}^{N}\right|_{\psi_{q}\left(U_{q}\right)}\right)}\right.$.

If these mappings are homeomorphisms, then the set $\mathcal{A}$, with

$$
\mathcal{A}=\left\{\left(U_{p}, \psi_{p}\right),\left(U_{q}, \psi_{q}\right)\right\}
$$

is called an atlas, with $\psi_{p}, \psi_{q}$ called coordinates.

Our interest is in one of the charts mapping equivalence classes. That is,

$$
\mathcal{A}=\left\{\left(\left[U_{p}\right],\left[\psi_{p}\right]\right),\left(U_{q}, \psi_{q}\right)\right\} .
$$

Similarly, for manifolds in derivatives of $\psi$, we get the atlases

$$
\mathcal{A}^{(i)}=\left\{\left(\left[U_{p}\right],\left[\psi_{p}^{(i)}\right]\right),\left(U_{q}, \psi_{q}^{(i)}\right)\right\} .
$$

$$
\text { 1) }
$$

Transmission mappings: The mapping from $\left.\left(\mathbb{R},\left.\mathcal{J}_{\mathbb{R}}\right|_{\left[\psi\left(\left[U_{p}\right]\right)\right.}\right]\right)$ to $\left(\mathbb{R},\left.\mathcal{J}_{\mathbb{R}}\right|_{\psi\left(U_{q}\right)}\right)$, having stepped down from $\mathbb{R}^{N}$ to $\mathbb{R}$, is given by

$$
\psi_{p}\left(\psi_{q}^{-1}\left(\psi_{q}\left(\left[U_{p}\right]\right)\right)\right)
$$

and it is called a transition mapping. Its inverse is

$$
\psi_{q}\left(\psi_{p}^{-1}\left(\psi_{p}\left(U_{q}\right)\right)\right)
$$

We are interested in case where $\left[U_{p}\right]$ and $U_{q}$ overlap, so that there is a point $x$ in the neighborhood of both $p$ and $q$ such that

$$
[\psi[x]]=\psi(x) .
$$

The transmission mappings in derivative spaces lead to

$$
\frac{d^{n}[\psi[x]]}{d x^{n}}=\frac{d^{n} \psi(x)}{d x^{n}},
$$

for $n=1,2,3, \cdots$.

2) Tangent Spaces: As indicated earlier, tangent spaces assist in establishing a function $f$, that allows for results to be projected onto the metric space.

A tangent space is a set

$$
\mathcal{T} \mathcal{P}=\left\{\mathcal{V}_{\gamma, P} \mid \gamma: \mathbb{R} \rightarrow X\right\},
$$

such that

$$
V_{\gamma, P} f=\left(f \circ \gamma^{-1}\right)\left[\gamma\left(\tau_{0}\right)\right]
$$

where $f \in C^{\infty}(X), \mathcal{V}_{\gamma, P}: C^{\infty}(\mathcal{M}) \rightarrow \mathbb{R}, \gamma\left(\tau_{0}\right)=P$.

The tangent space $\mathcal{T} \mathcal{P}$ has the basis vectors $\left\{\partial X^{i}\right\}$. Any vector then can be represented in terms of it, so that

$$
X=\left.\xi^{i} \frac{\partial}{\partial X^{i}}\right|_{P} .
$$

That is $X \in \mathcal{T}_{P} X=\mathcal{T}_{P} \mathcal{M}$.
3) Cotangent Spaces: A tangent space is a vector space, and where there is one there should also be a co-vector space, hence the cotangent space. It is the set of all maps in the tangent space to $\mathbb{R}$. That is,

$$
\omega: \mathcal{T}_{p} X \rightarrow \mathbb{R},
$$

with $\omega$ being an element of the cotangent space. The symbol $(d f)_{p}$ represents a co-vector acting on mapping $f$ at $P$. A cotangent space, therefore, is

$$
\mathcal{T P}^{*}=\left\{(d f)_{p} \mid f \in C^{\infty}(X)\right\},
$$

and it is a vectors space, and is the dual of $\mathcal{T} \mathcal{P}$.

The basis vectors of a cotangent space requires that

$$
\left.\left(d \omega^{j}\right)_{p}\left(\frac{\partial}{\partial x^{i}}\right)\right|_{p}=\delta_{i}^{j},
$$

so that

$$
\operatorname{Basis}\left(\mathcal{T P}^{*}\right)=\left.\left\{\frac{\partial}{\partial x^{i}}\right\}\right|_{p}
$$

Therefore an element $\omega$ of $\mathcal{T} \mathcal{P}^{*}$ can be written

$$
\omega=\left.\omega_{i}\left(d x^{i}\right)\right|_{p} .
$$

4) Quotient Spaces: Consider the general ordinary differential equation

$$
f\left(x, \psi, \psi^{\prime}, \psi^{\prime \prime}, \psi^{(3)}, \cdots\right)
$$

with

$$
\psi: X \rightarrow Y
$$

A set

$$
S=\left\{x_{0}, x_{1}, x_{2}, \cdots\right\} \subset X,
$$

such that

$$
x_{i}=P\left(x_{j}\right)=x_{j}+2 \pi k_{s}
$$

where $k_{s}$ is an integer, is called an equivalence class. This leads to an Quotient space $\mathbb{R} / \sim$. It is the set of all equivalent classes in $\mathbb{R}$, and is given by

$$
\mathbb{R} / \sim=\left\{\left[x_{0}\right],\left[x_{1}\right],\left[x_{2}\right], \cdots\right\} .
$$

It is a differentiable topological space. In our study, the image of this set, is also an equivalence class

$$
\left\{\left[\psi\left(x_{0}\right)\right],\left[\psi\left(x_{1}\right)\right],\left[\psi\left(x_{2}\right)\right], \cdots\right\},
$$

as such there is a homomorphism, and it extends to the derivative spaces

$$
\left\{\left[\psi^{(i)}\left(x_{0}\right)\right],\left[\psi^{(i)}\left(x_{1}\right)\right],\left[\psi^{(i)}\left(x_{2}\right)\right], \cdots\right\},
$$

for $i=1,2,3, \cdots$.

\section{THE FREE PARTICLE WAVE EQUATION (4)}

Equation (4) can be simplified by letting $t \rightarrow \frac{2 i m_{p}}{\hbar} \tau$. This reduces it to

$$
\frac{\partial^{2} \varphi}{\partial X^{2}}=\frac{\partial \varphi}{\partial \tau}
$$




\section{A. The pure Lie approach to (30)}

A treatment of (30) through symmetry methods can be found, almost, in all texts on the subject. The method were introduced by Sophus Lie (1842 - 1899), and continues to grow, see [1] and [2]. The use of the methods on the Schrödinger equation is not new, see [3] and [4].

To apply Lie's theory to equation (30), we seek to construct the transformations

$$
\begin{aligned}
\bar{\tau} & \approx \tau+\epsilon \xi^{1}(\tau, X, \varphi) \\
\bar{X} & \approx X+\epsilon \xi^{2}(\tau, X, \varphi) \\
\bar{\varphi} & \approx \varphi+\epsilon \eta(\tau, X, \varphi)
\end{aligned}
$$

with

$$
G=\xi^{1}(\tau, X, \varphi) \frac{\partial}{\partial \tau}+\xi^{2}(\tau, X, \varphi) \frac{\partial}{\partial X}+\eta(\tau, X, \varphi) \frac{\partial}{\partial \varphi},
$$

called the symmetry generator. The function $\eta$ can be simplified in advance as $\eta=f \varphi+g$, with $f=f(\tau, x)$ and $g=g(\tau, x)$, so that

$$
G=\xi^{1} \frac{\partial}{\partial \tau}+\xi^{2} \frac{\partial}{\partial X}+(f \varphi+g) \frac{\partial}{\partial \varphi},
$$

with $\xi^{1}=\xi^{1}(\tau, X, \varphi), \xi^{2}=\xi^{2}(\tau, X, \varphi)$ and $\eta=\eta(\tau, X, \varphi)$. The second order symmetry generator $G^{[2]}$ satisfies the invariance condition

$$
\left.G^{[2]}\left(\varphi_{X X}-\varphi_{\tau}\right)\right|_{\varphi_{X X}=\varphi_{\tau}}=0 .
$$

We use the notation $h_{s^{1} \cdots s^{n}}=\frac{\partial^{n} h}{\partial s^{1} \cdots \partial s^{n}}$, alternatively. The analysis of the expanded form of this invariance lead to the following Lie symmetries.

$$
\begin{aligned}
G_{1} & =\frac{\tau^{2}}{2} \frac{\partial}{\partial \tau}+\frac{X \tau}{2} \frac{\partial}{\partial X}-\left(\frac{X^{2}}{8}+\frac{\tau}{4}\right) \varphi \frac{\partial}{\partial \varphi} \\
G_{2} & =\tau \frac{\partial}{\partial \tau}+\frac{X}{2} \frac{\partial}{\partial X} \\
G_{3} & =\frac{\partial}{\partial \tau} \\
G_{4} & =\tau \frac{\partial}{\partial X}-\frac{X \varphi}{2} \frac{\partial}{\partial \varphi} \\
G_{5} & =\frac{\partial}{\partial X} \\
G_{6} & =\varphi \frac{\partial}{\partial \varphi} \\
G_{\infty} & =g \frac{\partial}{\partial \varphi}
\end{aligned}
$$

The symmetry $G_{\infty}$ is called the infinite symmetry, because $g$ is not determined.

\section{B. The solution through $G_{1}$}

The only solution that is widely used in application is through the symmetry $G_{1}$. It assumes the form

$$
\varphi=\left(D_{1} \frac{X}{\tau}+D_{2}\right) \frac{e^{-\frac{X^{2}}{4 \tau}}}{\sqrt{\tau}}
$$

where $D_{1}$ and $D_{2}$ are constants of integration. That is,

$$
\varphi=\left(D_{1} \frac{X}{\frac{\hbar}{2 i m_{p}} t}+D_{2}\right) \frac{e^{-\frac{X^{2}}{4 \frac{\hbar}{2 i m_{p}} t}}}{\sqrt{\frac{\hbar}{2 i m_{p}} t}} .
$$

From Figure 1, it is clear that (45) does not exhibit any quantum superpositions, which is unfortunate, because it is an experimental feature, observed in the physics of subatomic particles. This is a precursor to modified Lie symmetry analysis.

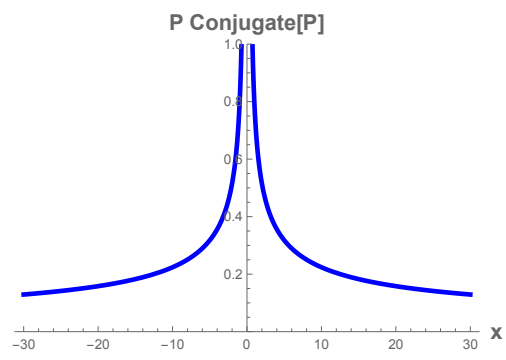

Fig. 1. A plot of the solution in (45), with $D_{1}=D_{2}=1$, and $T=1$.

\section{The modified Lie symmetry approach}

Definition 1: Suppose $G$ is a set of local point transformations from $\mathbb{R}^{N}$ to $\mathbb{R}^{N}$, and $\bar{G}$ is a set of local point transformations from $\mathbb{R}^{N}$ to $\tilde{\mathbb{R}}^{N}$. Then we call the transformations $\tilde{G}$, from $\mathbb{R}^{N}$ to $\tilde{\mathbb{R}}^{N}$, modified local transformations, if they are invertible.

From this follows the following theorem:

Theorem 1: If $\xi^{i}$ and $\tilde{\xi}^{i}$ are the Lie and modified infinitesimals of the same equation, then

$$
\left.\left(\bar{D}_{j} \bar{\xi}^{i}-D_{j} \xi^{i}\right)\right|_{\omega=0}=0, \quad i, j=1,2,3, \ldots,
$$

where $\omega$ is the parameter for modified symmetries.

Here $\bar{D}_{j}$ and $D_{j}$ are the operators of total differentiation, in their respective spaces. We use it to determine the modified symmetries from Lie's symmetries, one of which is

$$
\begin{aligned}
\tilde{G}_{1}= & \frac{2 e^{\omega^{2} X}}{\omega^{4}\left(\omega^{2}-1\right)} \cos (\omega \tau / i) \frac{\partial}{\partial X}+\frac{i e^{\omega^{2} X}}{\omega^{3}\left(\omega^{2}-1\right)} \\
& \sin (\omega \tau / i) \frac{\partial}{\partial \tau}-\frac{e^{\omega^{2} X}}{2} \cos (\omega \tau / i) \varphi \frac{\partial}{\partial \varphi},
\end{aligned}
$$

from which we get the characteristic equation

$\frac{\omega^{4}\left(\omega^{2}-1\right) e^{-\omega^{2} X} d X}{2 \cos (\omega \tau / i)}=\frac{i \omega^{3}\left(\omega^{2}-1\right) e^{-\omega^{2} X} d \tau}{\sin (\omega \tau / i)}=\frac{2 e^{-\omega^{2} X} d u}{\cos (\omega \tau / i) u}$.

Consequently, the transforming variables follow from

$$
\frac{\omega^{4}\left(\omega^{2}-1\right) e^{-\omega^{2} X} d X}{2 \cos (\omega \tau / i)}=\frac{i \omega^{3}\left(\omega^{2}-1\right) e^{-\omega^{2} X} d \tau}{\sin (\omega \tau / i)}
$$

and

$$
\frac{\omega^{4}\left(\omega^{2}-1\right) e^{-\omega^{2} X} d X}{2 \cos (\omega \tau / i)}=\frac{2 e^{-\omega^{2} X} d u}{\cos (\omega \tau / i) u}
$$

From (49), we have

$$
\frac{\omega^{2} d X}{2}=-\frac{\omega \cos (\omega \tau / i) d \tau}{i \sin (\omega \tau / i)}
$$


That is,

$$
\sigma=\frac{e^{-\omega^{2} X / 2}}{\sin (\omega \tau / i)}
$$

From (50), we have

$$
\frac{2 d u}{u}=\frac{\omega^{4}\left(\omega^{2}-1\right) d X}{2}
$$

so that

$$
\phi=u e^{\frac{-\omega^{4}\left(\omega^{2}-1\right) X}{4}},
$$

with $\phi=\phi(\sigma)$. Eventually, these transform (30) to

$$
2 \ddot{\phi}\left(e^{\omega^{2} X}-\sigma^{2}\right)+\frac{\sigma}{2} \dot{\phi}-\frac{\omega^{2}\left(\omega^{2}-1\right)}{4} \phi=0 .
$$

After a long analysis, we get

$$
\varphi=\left(D_{2}+D_{1} \frac{\tau}{X^{3 / 2}}\right) e^{\frac{-\tau^{2}}{4 X}}
$$

Reverting to the original variables, $\tau \rightarrow \frac{\hbar}{2 i m_{p}} t$, we have

$$
\varphi=\left(D_{2}+D_{1} \frac{\left(\frac{\hbar}{2 i m_{p}} t\right)}{X^{3 / 2}}\right) e^{\frac{-\left(\frac{\hbar}{2 i m_{p}} t\right)^{2}}{4 X}} .
$$

Since the particle is in motion, $\mid X>=c t$, with $c$ being its speed, we can have $t=X / c$, or $X=c\left(\frac{2 i m_{p}}{\hbar} \tau\right)$ and $t=$ $\left(\frac{\hbar}{2 i m_{p}}\right) X / c$ This suggests (30) can be written in the form

$$
\frac{\partial^{2} \varphi}{\partial\left(c \frac{2 i m_{p}}{\hbar} \tau\right)^{2}}=\frac{\partial \varphi}{\partial\left(\frac{\hbar}{2 i m_{p}} X / c\right)}
$$

or

$$
\frac{\partial^{2} \varphi}{\partial \tau^{2}}=\left(\frac{2 i m_{p} c}{\hbar}\right)^{3} \frac{\partial \varphi}{\partial X}
$$

Hence,

$$
\varphi=\left(D_{2}+D_{1} \frac{\left(\frac{\hbar}{2 i m_{p}} X / c\right)}{(c t)^{3 / 2}}\right) e^{\frac{-\left(\frac{\hbar}{2 i m_{p}} X / c\right)^{2}}{4 c t}} .
$$

A plot similar to the one in Figure 1 is then

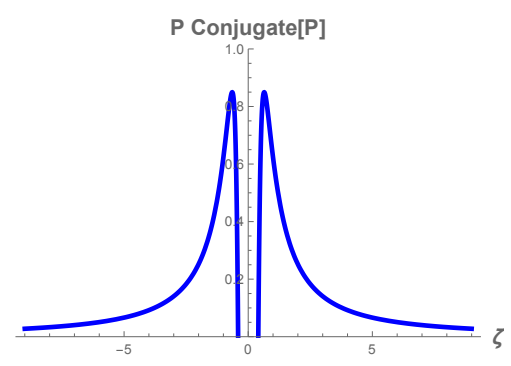

Fig. 2. A plot of the solution in (60), with $D_{1}=D_{2}=1$, and $T=1$. This plot agrees with the quantum superposition principle: The particle is at two positions at the same time.

The plot also suggests, in the transition from (57) to (60), what the quantum principle states about simultaneity of position, can also be said about time.

\section{A SIMPLIFICATION TO NEWTONIAN MECHANICS}

Anyone who has ever attempted to use group invariant solutions in practical applications, can attest how difficult it is to do so, primarily because of the unintegrable results the pure Lie approach usually leads to.

In here, we introduce a parameter $\omega$ in addition to $\epsilon$. The parameter $\epsilon$ maintains the role it has always been used for. The new parameter $\omega$ is used to evaluate the integrals, possible through limits and continuity principles.

\section{A. Gravity related radiation}

We are of the view that while Newton's mechanics has been successfully applied to a wide variety of areas, it is this wide variety that makes it difficult to understand $G$ through it. Newton's mechanics can be used to accurately predict the motions of satellites. For us, this mechanics does not really explain how they move. For a solid object in motion without spin, Newton's mechanics is usually used to describe the average motion. The interest is not on the motion of the individual atoms. While this is understandable, it would not be wise to account for the motion of the individual atoms in a satellite or a moving truck, but the sacrifice is huge. The true picture gets forgotten, and is replaced by Newton's averages. It then becomes impossible to explain concepts like $G$.

\section{B. Formulation of the model}

We are not really interested in exactly how this motion happens. There are just too many atoms in an object to account for each. We just want enough information that would allow a formula for $G$ to emerge. Applying Newton's mechanics to a single atom does not really help much. Applying it to the charges in the atom does make a difference; $G$ emerges. The simplest atom is the element Hydrogen. We only have two objects to work with. It is possible to go beyond this level, but that would generate too much information, more than what we bargained for. We would be overwhelmed.

In his universal gravitation law, Newton maintained that every particle of matter in the universe attracts every other particle with a force that is directly proportional to the product of the masses of the particles and inversely proportinal to the square of the distance between them. That is,

$$
\mathbf{F}_{N}=-G_{N} \frac{m_{1} m_{2}}{s^{2}} \hat{\mathbf{d}}_{1}
$$

This is called the universal gravitation law, and $G$ is the parameter whose formula we are interested in. Applied to two Hydrogen atoms of mass $m_{H}=m_{p}+m_{e}$ each, separated by the distance $r$, we have

$$
\mathbf{F}_{N}=-G_{N}\left(\frac{m_{H}}{s}\right)^{2} \hat{\mathbf{d}}_{1} .
$$

This was viewed as a two body problem, in that there are two bodies, one has mass $m_{1}$ and the other $m_{2}$, much like the Earth and the Moon. Our quest here is to understand the constant $G$ in the law, and this two body does not help us much in this regard. For us, this should be seen as a many body problem, in that the Earth has infinitely many atoms, and so does the Moon. This complicated picture can be simplified 
by considering the gravitational force between two hydrogen atoms. This is a four body problem, in that each atom has two subatomic particles, each with its own mass. The atom on its own is a two body problem.

1) The two-body problem for a Hydrogen atom : Let us consider a system consisting of two bodies of mass $m_{p}$ and $m_{e}$ at distances $\mathbf{r}_{p}$ and $\mathbf{r}_{e}$ from the galactic centre $O$. Let $\mathbf{F}_{p}$ and $\mathbf{F}_{e}$ be the external forces acting on $m_{p}$ and $m_{e}$, respectively, while $\mathbf{F}_{p e}$ is the internal force acting on body $m_{p}$ due to $m_{e}$, and $\mathbf{F}_{e p}$ the internal force acting on body $m_{e}$ due to $m_{p}$.

According to Newton's second law, the motionof the two bodies may be written as

$$
m_{p} \frac{d^{2} \mathbf{r}_{p}}{d t^{2}}=\mathbf{F}_{p}+\mathbf{F}_{p e}
$$

and

$$
m_{e} \frac{d^{2} \mathbf{r}_{e}}{d t^{2}}=\mathbf{F}_{e}+\mathbf{F}_{e p}
$$

The centre of mass coordinate $\mathbf{R}$ is given by

$$
\mathbf{R}=\frac{m_{p} \mathbf{r}_{p}+m_{e} \mathbf{r}_{e}}{m_{p}+m_{e}},
$$

and the relative coordinate $\mathbf{r}$ is given by

$$
\mathbf{r}=\mathbf{r}_{p}-\mathbf{r}_{e}
$$

The inverse transformation are given by

$$
\mathbf{r}_{e}=\mathbf{R}+\frac{m_{e}}{m_{p}+m_{e}} \mathbf{r}
$$

and

$$
\mathbf{r}_{p}=\mathbf{R}-\frac{m_{p}}{m_{p}+m_{e}} \mathbf{r}
$$

To transform the equations from $\mathbf{r}_{p}$ and $\mathbf{r}_{e}$ to $\mathbf{R}$, we add (63) and (64); that is

$$
m_{p} \frac{d^{2} \mathbf{r}_{p}}{d t^{2}}+m_{e} \frac{d^{2} \mathbf{r}_{e}}{d t^{2}}=\mathbf{F}_{p}+\mathbf{F}_{p e}+\mathbf{F}_{e}+\mathbf{F}_{e p} .
$$

According to Newton's third law

$$
\mathbf{F}_{p e}+\mathbf{F}_{e p}=0
$$

hence,

$$
\frac{d^{2}\left(m_{p} \mathbf{r}_{p}+m_{e} \mathbf{r}_{e}\right)}{d t^{2}}=\mathbf{F}_{p}+\mathbf{F}_{e}
$$

Using the result in (65), the preceding equation may be written as

$$
\left(m_{p}+m_{e}\right) \frac{d^{2} \mathbf{R}}{d t^{2}}=\mathbf{F}
$$

Hence,

$$
M \frac{d^{2} \mathbf{R}}{d t^{2}}=\mathbf{F}
$$

where $\mathbf{F}=\mathbf{F}_{p}+\mathbf{F}_{e}$, and $M=m_{p}+m_{e}$. This is the first of the equations we are interested in. To get the second, we multiply (63) by $m_{e}$ and (64) by $m_{p}$ and subtract:

$$
\begin{aligned}
& m_{e} m_{p} \frac{d^{2} \mathbf{r}_{p}}{d t^{2}}-m_{p} m_{e} \frac{d^{2} \mathbf{r}_{e}}{d t^{2}}=m_{e} \mathbf{F}_{p}+m_{e} \mathbf{F}_{p e} \\
& -m_{p} \mathbf{F}_{e}-m_{p} \mathbf{F}_{e p} .
\end{aligned}
$$

It can be rewritten as This is the first of the equations we are interested in. To get the second, we multiply (63) by $m_{e}$ and (63) by $m_{p}$ and subtract:

$m_{e} m_{p} \frac{d^{2}\left(\mathbf{r}_{p}-\mathbf{r}_{e}\right)}{d t^{2}}=m_{e} \mathbf{F}_{p}-m_{p} \mathbf{F}_{e}+\left(m_{e}+m_{p}\right) \mathbf{F}_{p e}$

Dividing by $m_{e}+m_{p}$ and using the result in (66):

$$
\frac{m_{e} m_{p}}{m_{e}+m_{p}} \frac{d^{2} \mathbf{r}}{d t^{2}}=\frac{1}{m_{e}+m_{p}}\left(m_{e} \mathbf{F}_{p}-m_{p} \mathbf{F}_{e}\right)+\mathbf{F}_{p e}
$$

Introducing the quantity $\mu$, called the reduced mass, and defined by

$$
\mu=\frac{m_{e} m_{p}}{m_{e}+m_{p}}
$$

reduces the preceding equation into

$$
\mu \frac{d^{2} \mathbf{r}}{d t^{2}}=\frac{m_{e} \mathbf{F}_{p}-m_{p} \mathbf{F}_{e}}{m_{e}+m_{p}}+\mathbf{F}_{p e}
$$

\section{Our magnetism based gravity}

Our hypothesis is that an atom has a velocity relative to the centre of the universe; each individual charge in the atom then has a magnetic field; the nett sum of these fields at any point is not zero. This non-zero sum is what we believe is the source of gravity. We will now build this up for two Hydrogen atoms, a distance $r$ apart.

Let $\mathbf{r}_{p}$ be the position vector relative to the centre of the universe for a proton in a Hydrogen atom. The velocity is then $\dot{\mathbf{r}}_{p}$. Its magnetic field $\mathbf{B}_{p b}$ at position $\mathbf{a}_{1}$, occupied by a proton of another Hydrogen, is then

$$
\mathbf{B}_{p, p}=\frac{\mu_{0}}{4 \pi} e \dot{\mathbf{r}}_{p} \times \frac{\mathbf{d}_{p, p}}{d_{p, p}^{3}} .
$$

Magnetic field of the electron at the same point is

$$
\mathbf{B}_{e, p}=-\frac{\mu_{0}}{4 \pi} e \dot{\mathbf{r}}_{e} \times \frac{\mathbf{d}_{e, p}}{d_{e, p}^{3}} .
$$

The sum of the two gives

$$
\mathbf{B}=\frac{\mu_{0}}{4 \pi} e\left(\dot{\mathbf{r}}_{p} \times \frac{\mathbf{d}_{p, p}}{d_{p, p}^{3}}-\dot{\mathbf{r}}_{e} \times \frac{\mathbf{d}_{e, p}}{d_{e, p}^{3}}\right),
$$

or

$$
\mathbf{B}=\frac{\mu_{0}}{4 \pi} e\left(\left[\dot{\mathbf{r}}_{e}+\mathbf{u}\right] \times \frac{\mathbf{d}_{p, p}}{d_{p, p}^{3}}-\dot{\mathbf{r}}_{e} \times \frac{\mathbf{d}_{e, p}}{d_{e, p}^{3}}\right) .
$$

That is,

$$
\mathbf{B}=\frac{\mu_{0}}{4 \pi} e\left(\mathbf{u} \times \frac{\mathbf{d}_{p, p}}{d_{p, p}^{3}}+\dot{\mathbf{r}}_{e} \times\left(\frac{\mathbf{d}_{p, p}}{d_{p, p}^{3}}-\frac{\mathbf{d}_{e, p}}{d_{e, p}^{3}}\right)\right),
$$

where $\mathbf{u}=\dot{\mathbf{r}}_{p}-\dot{\mathbf{r}}_{e}$.

From the Biot-Savart law:

$$
\mathbf{F}=q \mathbf{v} \times \mathbf{B},
$$

the force on the second proton moving with velocity $\mathbf{v}$ is

$$
\begin{aligned}
& F_{p}=\frac{\mu_{0}}{4 \pi} e q \\
& \left(\mathbf{v} \times \mathbf{u} \times \frac{\mathbf{d}_{p, p}}{d_{p, p}^{3}}+\mathbf{v} \times \dot{\mathbf{r}}_{e} \times\left(\frac{\mathbf{d}_{p, p}}{d_{p, p}^{3}}-\frac{\mathbf{d}_{e, p}}{d_{e, p}^{3}}\right)\right) .
\end{aligned}
$$


The identity $\mathbf{a} \times \mathbf{b} \times \mathbf{c}=(\mathbf{a} \cdot \mathbf{c}) \mathbf{b}-(\mathbf{a} \cdot \mathbf{b}) \mathbf{c}$ reduces it to where

$$
\begin{array}{cc}
F_{p}=\frac{\mu_{0}}{4 \pi} \text { e } q & \tilde{\mu}=\frac{e^{2}}{4 \pi \epsilon_{0} \mu}, \\
\qquad\left(\left(\frac{\mathbf{d}_{p, p}}{d_{p, p}^{3}} \cdot \mathbf{v}\right) \mathbf{u}-(\mathbf{v} \cdot \mathbf{u}) \frac{\mathbf{d}_{p, p}}{d_{p, p}^{3}}+\mathbf{v} \times \dot{\mathbf{r}}_{e} \times\left(\frac{\mathbf{d}_{p, p}}{d_{p, p}^{3}}-\frac{\mathbf{d}_{e, p}}{d_{e, p}^{3}}\right)\right) . & v=\frac{d s}{d t},
\end{array}
$$

$$
\begin{aligned}
& F_{e}=\frac{\mu_{0}}{4 \pi} e q \\
& \left(\left(\frac{\mathbf{d}_{e, e}}{d_{e, e}^{3}} \cdot \mathbf{v}\right) \mathbf{u}-(\mathbf{v} \cdot \mathbf{u}) \frac{\mathbf{d}_{e, e}}{d_{e, e}^{3}}+\mathbf{v} \times \dot{\mathbf{r}}_{e} \times\left(\frac{\mathbf{d}_{e, e}}{d_{e, e}^{3}}-\frac{\mathbf{d}_{p, e}}{d_{p, e}^{3}}\right)\right) .
\end{aligned}
$$

and

This is our impression of a force between two electrically neutral objects.

It is assumed that the two Hydrogen atoms are moving at the same speed, and in the same direction, hence $u \cdot v=v^{2}$.

From $\mathbf{F}_{N}=\mathbf{F}_{E M}$, we have

$$
-G \frac{m_{1} m_{2}}{s^{2}} \hat{\mathbf{d}}_{1}=-\frac{\mu_{0}}{4 \pi}\left[\frac{e v}{s}\right]^{2} \hat{\mathbf{d}}_{1} .
$$

We replaced $G_{N}$ with $G$, just to able to compare because our $G$ is not really a constant, as equation (99) below suggests.

\section{Determining $v$}

It is assumed that the electron and proton are held together to form the hydrogen atom by some force $\mathbf{F}_{e}$, which could be described through Coulomb potential or the Yukawa potential. Such a system constitutes a two-body problem whose equations of motion are

$$
\begin{aligned}
& m_{p} \ddot{\mathbf{r}}_{1}=\mathbf{F}_{e}, \\
& m_{e} \ddot{\mathbf{r}}_{2}=-\mathbf{F}_{e}
\end{aligned}
$$

where $m_{p}$ and $m_{e}$ are masses for the proton and electron, respectively, and

$\mathbf{r}=\mathbf{r}_{1}-\mathbf{r}_{2}$. This system simplify to

$$
\begin{aligned}
M \ddot{\mathbf{R}} & =0, \\
\mu \ddot{\mathbf{r}} & =\mathbf{F}_{e},
\end{aligned}
$$

with $M=m_{p}+m_{e}$ and

$$
\mu=\frac{m_{p} m_{e}}{m_{p}+m_{e}}
$$

and

$$
F_{e}=-\frac{1}{4 \pi \epsilon_{0}}\left[\frac{e}{s}\right]^{2} .
$$

That is, to find $u$ and hence $G$, we have to solve the system constituted by

$$
\mu \frac{d^{2} s}{d t^{2}}=-\frac{1}{4 \pi \epsilon_{0}}\left[\frac{e}{s}\right]^{2}
$$

That is,

$$
\frac{d^{2} s}{d t^{2}}=-\frac{e^{2}}{4 \pi \epsilon_{0} \mu} \frac{1}{s^{2}}
$$

so that

$$
v \frac{d v}{d s}=-\frac{\tilde{\mu}}{s^{2}}
$$

$$
G=\frac{\mu_{0}}{4 \pi}\left[\frac{e v}{m_{H}}\right]^{2} .
$$

These can be put together to give

$$
\begin{aligned}
& G_{t t}-\left(\frac{2 \pi}{\mu_{0}}\right) \frac{G_{t}^{2}}{v} \\
& +\frac{\mu_{0}}{2 \pi}\left(\frac{e}{m_{H}}\right)^{2}\left(\frac{e^{2}}{4 \pi \epsilon} \frac{m_{p}+m_{e}}{m_{p} m_{e}}\right) \frac{1}{v}=0,
\end{aligned}
$$

with $G=G(t, s, v)$.

One other condition that $G$ has to satisfy is that gravity fields travel a the speed of light. Equation (95) solves into

$$
\frac{v^{2}}{2}+A=\frac{\tilde{\mu}}{s}
$$

and

$$
\begin{aligned}
& \frac{1}{v+\sqrt{-2 A}}+\frac{1}{v-\sqrt{-2 A}}+\frac{1}{\sqrt{-2 A}} \ln \left[\frac{v-\sqrt{-2 A}}{v+\sqrt{-2 A}}\right] \\
= & -\frac{e^{2}(2 A)}{4 \pi \epsilon_{0} \mu \tilde{\mu}^{2}} t+B
\end{aligned}
$$

where $A$ and $B$ are integration constants. That is,

$$
\begin{aligned}
& \frac{\sqrt{-2 A}}{v+\sqrt{-2 A}}+\frac{\sqrt{-2 A}}{v-\sqrt{-2 A}}+\ln \left[\frac{v-\sqrt{-2 A}}{v+\sqrt{-2 A}}\right] \\
= & -i \frac{e^{2}(2 A)^{3 / 2}}{4 \pi \epsilon_{0} \mu \tilde{\mu}^{2}} t+B \sqrt{-2 A} .
\end{aligned}
$$

It follows from this that the frequency $f$ is

$$
\begin{aligned}
f & =\frac{e^{2}(2 A)^{3 / 2}}{4 \pi \epsilon_{0} \mu \tilde{\mu}^{2}}=\frac{e^{2}(2 A)^{3 / 2}}{4 \pi \epsilon_{0} \mu} \frac{16 \pi^{2} \epsilon_{0}^{2} \mu^{2}}{e^{4}} \\
& =\frac{4 \pi \epsilon_{0} \mu(2 A)^{3 / 2}}{e^{2}}=\frac{4 \pi \epsilon_{0} \mu}{e^{2}}\left(\frac{\tilde{\mu}}{2 s_{0}}-v_{0}^{2}\right)^{3 / 2}
\end{aligned}
$$

The parameter $s_{0}$ represents the ground state of the Hydrogen atom, wherein the electron is moving at the speed $v_{0}$. This lead to tangential solutions

$$
v=V \cos (f t)
$$

and

$$
G=G_{N} \cos (f t),
$$

for some undetermined parameter $V$ and $G_{N}$, with $f$ given by

$$
f=\sqrt{\frac{\left(\frac{\frac{\tilde{\mu}}{s_{0}}-v_{0}^{2}}{2}\right)^{3}}{\mu^{2}}} .
$$


Substituting (105) into (99) leads to

$$
\begin{aligned}
& G_{N}= \\
& \left.\frac{i}{u Z_{H}\left(-K_{e} \frac{\left(e^{-}\right)^{2}}{9}\right)^{2}}\left(2 K_{v} \frac{\left(e^{-}\right)^{2}}{9}\left(\frac{v_{0}^{2}}{2}-\frac{\left(-K_{e} \frac{\left(e^{-}\right)^{2}}{9}\right)}{r_{0}}\right)\right)^{3}\right) \times \\
& \left(\frac{v_{0}^{2}\left(-K_{e} \frac{\left(e^{-}\right)^{2}}{9}\right)^{2}}{2\left(\frac{v_{0}^{2}}{2}-\frac{\left(-K_{e} \frac{\left(e^{-}\right)^{2}}{9}\right)}{r_{0}}\right)^{3}}+\left(s_{0}+\frac{e^{\frac{i \pi}{3}}\left(-K_{e} \frac{\left(e^{-}\right)^{2}}{9}\right)}{\frac{v_{0}^{2}}{2}-\frac{\left(-K_{e} \frac{\left(e^{-}\right)^{2}}{9}\right)}{s_{0}}}\right)^{2}\right),
\end{aligned}
$$

with the parameter $v_{0}$ is given by the quintic equation in $v_{0}^{2}$ :

$$
\alpha_{5} v_{0}^{10}+\alpha_{4} v_{0}^{8}+\alpha_{3} v_{0}^{6}+\alpha_{2} v_{0}^{4}+\alpha_{1} v_{0}^{2}+\alpha_{0}=0 .
$$

Its parameters are given by

$$
\begin{aligned}
\alpha_{0} & =-4 \mu_{0}^{4}, \\
\alpha_{1} & =8 s_{0} \mu_{0}^{3}, \\
\alpha_{2} & =-8 c^{2} s_{0} \mu_{0}^{3}, \\
\alpha_{3} & =12 c^{2} s_{0}^{2} \mu_{0}^{2}, \\
\alpha_{4} & =-6 c^{2} s_{0}^{3} \mu_{0}, \\
\alpha_{5} & =c^{2} s_{0}^{4} \mu_{0},
\end{aligned}
$$

and $\mu=-K_{e}\left(e^{-}\right)^{2} / 9$. with the physical parameters

$$
\begin{aligned}
& G=6.67259 * 10^{-11} \mathrm{Nm}^{2} / \mathrm{kg}^{2}, \\
& \text { gravitational constant from experiment, } \\
& s_{0}=\frac{1}{2}(0.52917720859) * \AA \text {, } \\
& \text { Bohr's atom size, } \\
& s_{0}=\frac{1}{2}(0.528) * \AA \text {, } \\
& \text { hypothetical atom size, } \\
& e^{-}=1.602176487 * 10^{-19} \mathrm{C} \text {, } \\
& \text { charge of an electron, } \\
& K_{v}=\frac{\mu_{0}}{4 \pi}=1 * 10^{-7} \mathrm{~Wb} /(\mathrm{A} \mathrm{m}) \text {, } \\
& \text { permeability of free space, } \\
& K_{e}=8.987551787 * 10^{9} \mathrm{~N} / \mathrm{C}^{2} \text {, } \\
& \text { permittivity of free space, } \\
& c=2.99792458 * 10^{8} \mathrm{~m} / \mathrm{s} \text {, } \\
& \text { speed of light in vacuum, } \\
& Z_{H}=1.000794 \text {, } \\
& \text { mass number of an Hydrogen atom, } \\
& u=1.660538782 * 10^{-27} \mathrm{~kg} \text {, } \\
& \text { atomic mass unit. }
\end{aligned}
$$

\section{E. A numerical value for $G$}

Solution of the quintic through numerical techniques yields ten roots. It is only two of them that lead to the correct answer Bohr's atom size gives

$$
v_{0}= \pm 6.89696 i * 10^{-10} \mathrm{~m} / \mathrm{s},
$$

from which the gravitational constant is found to be

$$
G_{N}=6.656 * 10^{-11} \mathrm{Nm}^{2} / \mathrm{kg}^{2},
$$

a $99.8 \%$ accuracy.

Our calculations suggest a Hydrogen atom size slightly larger by about $0.2 \%$ (what we refer to as the hypothetical atom size, above). It led to

$$
v_{0}= \pm 6.90451 i * 10^{-10} \mathrm{~m} / \mathrm{s},
$$

from which the gravitational constant is found to be

$$
G_{N}=6.671 * 10^{-11} \mathrm{Nm}^{2} / \mathrm{kg}^{2},
$$

a value that compares more favorably with the experimental result at $99.98 \%$.

The same effect can be realized by rounding off the Bohr obtained $v$ to $\pm 6.9 i * 10^{-10} \mathrm{~m} / \mathrm{s}$, thus leaving Bohr's model intact.

\section{CONCLUSION}

We tested our hypothesis on how matter generates gravity by determining a formula for $G$, the universal gravitational constant. In the process, we noted that gravitation has a frequency. And that this frequency is uniquely associated with the hydrogen atom that we used here as an example. From this we deduce that this frequency need not be the same for all material elements, as some purported.

The frequencies can assist in identifying and harvesting the vast amount of free energy, that is all over the universe, around us and even permeating through us. They can also be used for security purposes, like detecting prohibited substances. And also be used to determine material elements underneath without the need to draw a sample.

They can also be used militarily to detect and neutralize miniaturized offensive devices.

\section{REFERENCES}

[1] Gang wei Wang, Xi qiang Liu, and Ying yuan Zhang. Lie symmetry analysis to the time fractional generalized fifth-order kdv equation. Communications in Nonlinear Science and Numerical Simulation, 18:23212326, 92013.

[2] Taha Aziz, F. M. Mahomed, and D. P. Mason. A unified compatibility method for exact solutions of non-linear flow models of newtonian and non-newtonian fluids. International Journal of Non-Linear Mechanics, 78:142-155, 12016.

[3] Michael Martin Nieto and D Rodney Truax. Symmetries and solutions of the three-dimensional paul trap. 2001.

[4] Matthew Robinson. Symmetry and the Standard Model Mathematics and Particle Physics. Springer, 2011.

\section{Creative Commons Attribution License 4.0} (Attribution 4.0 International, CC BY 4.0)

This article is published under the terms of the Creative Commons Attribution License 4.0

https://creativecommons.org/licenses/by/4.0/deed.en_US 